Jeux de rôle néomédiévaliste et rhétorique de la démédiation : les procédés d'ouverture et de fermeture des hétérotopies goréennes dans Second Life

Neo-medieval role-plays and the rethoric of de-mediation: the opening and closing processes of Gorean heterotopies in Second Life

\title{
Christophe Duret
}

\section{CpenEdition}

Journals

Édition électronique

URL : http://journals.openedition.org/communicationorganisation/5059

DOI : 10.4000/communicationorganisation.5059

ISSN : 1775-3546

Éditeur

Presses universitaires de Bordeaux

Édition imprimée

Date de publication : 1 décembre 2015

Pagination : 49-60

ISSN : 1168-5549

Référence électronique

Christophe Duret, « Jeux de rôle néomédiévaliste et rhétorique de la démédiation : les procédés d'ouverture et de fermeture des hétérotopies goréennes dans Second Life », Communication et organisation [En ligne], 48 | 2015, mis en ligne le 01 décembre 2018, consulté le 03 janvier 2020. URL http://journals.openedition.org/communicationorganisation/5059; DOI : 10.4000/ communicationorganisation.5059 


\title{
Jeux de rôle néomédiévalistes et rhétorique de la démédiation : les procédés d'ouverture et de fermeture des hétérotopies goréennes dans Second Life
}

\author{
Christophe Duret $^{1}$
}

\section{Introduction}

Les utopies proposées dans les jeux vidéo, affirme Schulzke (2014), n'offriraient aucune perspective critique sur la société, se contentant d'en proposer une version idéalisée sans remettre en question son statu quo. Ce serait à travers les dystopies, selon lui, que les jeux vidéo apporteraient une réelle perspective critique en raison de leur capacité à dépeindre les conséquences potentielles de problèmes sociaux réels non réglés et à ouvrir un espace de contestation. La portée critique des dystopies vidéoludiques paraît indéniable. Toutefois, nous refusons de dénier aux utopies vidéoludiques cette caractéristique. Si les dystopies constituent des projections sur le mode de l'exacerbation de tendances indésirables décelées dans un état de société donné, les utopies constituent pour leur part des projections alternatives et imaginaires dans lesquelles ces tendances sont évacuées. Il ne s'agit donc pas de dépeindre le monde réel sous un jour idéal sans remettre en cause son statu quo, contrairement à ce qu'écrit Schulzke, mais de construire une société sur de nouvelles fondations, dont les composantes à l'origine dudit statu quo sont absentes. Toutefois, la portée critique de l'utopie se présente en creux, sur un mode passif. Les utopies vidéoludiques remplissent également une fonction critique - active, cette fois-ci - lorsque, réalisées par une communauté de joueurs dans un monde virtuel, elles deviennent des hétérotopies. C'est ce que nous verrons dans le cadre de cet article avec le cas des jeux de rôle goréens. Plus précisément, nous nous interrogerons sur les modalités à l'aide desquelles ces jeux traduisent, dans une double logique hétérotopique et vidéoludique, un désir utopique nostalgique : le fantasme de démédiation. Nous décrirons donc

1 Christophe Duret détient une Maîtrise en communication. Il est doctorant en études françaises à l'Université de Sherbrooke depuis 2014 et gère les Éditions de l'Inframince. Il codirige actuellement un ouvrage collectif sur l'intertextualité dans les jeux vidéo. Son champ d'intérêt en recherche porte sur les jeux de rôle en ligne, qu'il aborde dans une double perspective sociocritique et herméneutique ; Christophe.Duret@usherbrooke.ca 
sommairement ce fantasme, pour définir ensuite le concept d'hétérotopie. Nous nous intéresserons enfin au système d'ouverture et de fermeture à l'aide duquel les hétérotopies remplissent les fonctions de compensation et de critique, de même qu'à ses caractéristiques, que nous décrirons en tant que modes de mise en relation, de mise en coprésence et de circonscription.

Bien qu'il constitue pour l'essentiel une proposition théorique, cet article repose néanmoins sur une analyse des documents produits par des membres de la communauté goréenne de Second Life témoignant indirectement de leurs pratiques vidéoludiques. Nous avons travaillé à partir d'un corpus de près de 5000 billets répartis sur 70 fils de discussion, publiés sur les forums de discussion The Gorean Forums et Gor-SL. Nous avons également analysé des blogues de joueurs, des captures d'écran et des captures vidéo de séances de jeu ainsi que des encyclopédies en ligne et des revues amateur consacrés aux jeux goréens et aux romans dont ils s'inspirent. Nous nous sommes enfin adonnés à de l'observation participante entre l'été 2012 et aujourd'hui.

\section{Une utopie nostalgique}

Le mot utopie est forgé par Thomas More à partir du privatif grec ov̉ et de $\tau$ 七o $\pi$ s, lieu, signifiant étymologiquement " en aucun lieu ». Il apparaît pour la première fois dans son optimo rei publicae statu, deque nova insula Utopia, désignant l'île imaginaire d'Utopia où se trouve une société idéale réalisant le bonheur de chacun. Le terme évolue sémantiquement, entre le XVI et le $\mathrm{XIX}^{\mathrm{e}}$ siècle. Il désigne d'abord un lieu ou un pays imaginaire, puis un genre littéraire proposant des plans de gouvernements imaginaires, avant de devenir une expression adressée péjorativement aux réformistes dont le programme politique est jugé irréaliste puis, avant la Révolution de 1848, un synonyme de socialisme et de communisme (Funcke 1988).

Selon Funcke (1988), l'étymologie d'Utopia aurait dû être $a$-topia et non ou-topia, mais le ou permettait à Thomas More de jouer sur l'ambiguïté des mots outopia et eutopia (ce dernier étant créé à partir de $\varepsilon \hat{v}$, «bon », soit un lieu bon, où tout est au mieux), entre un lieu qui n'existe en aucun lieu et un lieu bon, où tout est au mieux, « ou » et « eu » étant homophones pour un locuteur anglais. Une telle ambiguité rend compte de la double nature de l'utopie. Lieu situé hors du réel, il offre à l'utopiste la distance nécessaire à l'appréciation du monde qu'il habite. Lieu favorisant le bonheur de tous, il constitue un idéal, mais aussi une proposition pour améliorer le monde réel.

Si depuis L'An 2440, de Louis-Sébastien Mercier, nombre d'utopies posent leur regard sur l'avenir, rien ne s'oppose, néanmoins, à ce qu'elles se tournent vers le passé. Ainsi en est-il du jeu en ligne World of Warcraft qui, selon Galloway (2006), se présente comme une utopie précapitaliste. Ainsi en est-il également des mythes de l'Arcadie et de l'Âge d'or. Dès lors, les principes appréciatif et amélioratif de l'utopie s'appuient sur un sentiment de nostalgie, ce désir de retrouver un temps révolu et idéalisé. 
C'est à cette forme d'utopie nostalgique qu'un grand nombre d'œuvres d'inspiration médiévale correspondent lorsqu'elles opposent terme à terme un goût pour un passé idyllique et le rejet d'un présent dysphorique (Duret 2014a). Cette opposition de fonde sur la polarisation suivante : rapport direct de l'homme médiéval à la nature, liens conviviaux et immédiats avec son prochain, structure sociale stable, claire et hiérarchisée, d'un côté. De l'autre côté figure l'homme contemporain dans son rapport aliéné à l'environnement et à une société complexe, ambiguë, abstraite, déshumanisée et sur laquelle il est sans emprise. Cette polarisation traduit un phénomène que nous avons qualité de fantasme de " démédiation " (Duret 2014a), une aspiration visant à connaître une expérience « démédiée » du monde, plus proche de la nature, moins filtrée sur les plans social et technique. Ce fantasme, qui s'exprime sous la forme d'une utopie nostalgique, est la traduction vidéoludique d'un rejet de ce que nous appelons, par soucis de symétrie, la surmédiation, un sentiment d'aliénation éprouvé devant la complexification et l'abstraction croissantes de lorganisation sociale contemporaine et ses dimensions constitutives (économique, médiatique, technologique, etc.) et devant les intermédiaires (dispositifs médiatiques et communicationnels, corps de lois, etc.) qui s'interposent entre les individus et dans la relation de ces derniers à leur environnement. Sous l'influence du fantasme de démédiation s'instituent des œuvres et des univers refuges qui donnent lieu à des utopies nostalgiques vues comme aspiration vers une condition prémoderne.

Les jeux de rôle goréens figurent parmi les œuvres où se traduit une telle aspiration, comme nous le verrons bientôt. Les jeux de rôle goréens sont des jeux de rôle participatifs en environnement virtuel organisés dans Second Life. Ils s'inspirent des Chroniques de Gor, un cycle de 33 romans écrits entre 1966 et 2013 par John Norman. Les Chroniques de Gor mêlent science-fiction et fantasy.

Les jeux de rôle participatifs en environnement virtuel (ci-après, les «JRPEV») ont lieu dans des environnements virtuels en ligne multi-usagers (ci-après, "MUVE ») tels que Second Life et IMVU, au sein desquels les joueurs, suivant une logique participative, conçoivent les décors, les dispositifs techniques (par exemple, les compteurs de points de vie), les règlements et les accessoires nécessaires à son fonctionnement.

La planète Gor, dépeinte dans les romans de Norman et servant de décor aux JRPEV goréens, est artificiellement maintenue dans un état de civilisation prémoderne inspiré de l'Antiquité et du Moyen Âge terriens en raison d'un interdit religieux qui y frappe le développement de la technologie. Les romans reposent sur une philosophie néo-darwinienne, l'ordre naturel. En vertu de cette philosophie règne la loi du plus fort, mais également un dimorphisme sexuel justifiant une dissymétrie entre les hommes et les femmes, les secondes s'épanouissant dans la soumission et la servitude et les premiers, étant naturellement dominants et occupant une position de maîtres. 
Les JRPEV goréens se déploient à travers un ensemble de "sims ", des espaces virtuels en trois dimensions loués par Second Life, aménagés par les joueurs et liés entre eux par des portails de téléportation. La communauté goréenne se divise grossièrement en deux sous-cultures, caractérisées par la posture interprétative des joueurs sur les Chroniques de Gor. D'une part, on y retrouve les joueurs qui se veulent fidèles aux romans de Norman dans leur jeu de rôle. Ils se qualifient de By-the-Books. D'autre part, il y a les joueurs qui se distancient du contenu des romans pour introduire en jeu des éléments exogènes. Ce sont les joueurs Gor Evolved. Les conflits entre les deux groupes concernent pour l'essentiel le rôle des femmes dans la société goréenne et la loi de l'ordre naturel. Les By-the-Books affirment que la femme n'est pas l'égale de l'homme, sur Gor, et refusent qu'elle occupe le rôle de guerrières, à l'inverse des Gor Evolved qui avalisent l'existence d'un tel rôle.

Nous avons vu ailleurs (Duret 2014a) l'adéquation de Gor au fantasme de démédiation. Nous la résumons ici. Dans les JRPEV goréens, il existe une intime interpénétration de la nature et de l'habitat. Le village est privilégié et la cité se maintient à une échelle humaine, gardant des proportions modestes. Une relation intime unit les Goréens à la nature : d'une part, l'ordre naturel établit le primat du biologique sur le culturel, d'autre part, l'économie archaïque de Gor repose sur la chasse, l'agriculture, l'élevage et l'artisanat, de sorte que les Goréens tirent de leur environnement les ressources nécessaires à leur subsistance, qu'ils transforment grâce aux compétences développées à son contact. Les relations sociales sont stables, prévisibles et hiérarchisées en vertu d'un système de castes au sein duquel le rôle de chacun et la nature des relations qui les unit aux autres sont explicites - ce qui prémunit le joueur d'un état de société complexe et ambigu. Gor constitue donc un monde plus proche de la nature et les relations qui se tissent entre les Goréens et avec leur environnement sont faiblement filtrées, médiées, aux plans social et technique. C'est en permettant la réalisation du fantasme de démédiation que Gor se qualifie en tant qu'utopie nostalgique, et ce à travers une perspective rétrogressive (Séguy 1971) sur les relations sociales, à commencer par les relations hommes-femmes. Gor répond ainsi au double principe appréciatif et amélioratif de toute utopie en critiquant la surmédiation contemporaine et en proposant un monde alternatif démédié.

\section{Des hétérotopies}

S'il est possible de distinguer les utopies écrites et les utopies pratiquées, les premières relevant de la fiction et les secondes témoignant d'une tentative historique d'application d'un projet utopique (Séguy 1971), la démarcation ne va pas de soi dans le cas des JRPEV goréens. D'un côté, l'élan utopique s'y exprime au sein d'une fiction (le monde de Gor comme traduction du fantasme de démédiation), signalant au passage son appartenance à une utopie écrite. De l'autre, les JRPEV goréens regroupent une communauté de 
joueurs qui adopte un mode de vie répondant à une utopie nostalgique dans un cadre prémoderne - bien que cette tentative ait lieu sur les modes de la fiction et de la simulation - relevant de la fantaisie et non d'une tentative de transformation historique. Il n'y a donc lieu de parler ni d'utopie écrite (ou fictionnelle) ni d'utopie pratiquée, mais d'hétérotopie vidéoludique.

\section{Espaces de localisation, de l'étendue, de l'emplacement}

Selon Foucault (1994), les utopies et les hétérotopies constituent des types spécifiques d'emplacements. Dans Des espaces autres, le philosophe brosse une histoire sommaire de l'espace en trois temps, du Moyen Âge à nos jours. L'espace médiéval est un ensemble de lieux nettement hiérarchisés. C'est un espace de localisation qui a été remplacé, au $\mathrm{XVII}^{\mathrm{e}}$ siècle, avec la révolution galiléenne, par un espace de l'étendue, ouvert à l'infini. L'espace contemporain serait celui de l'emplacement, un réseau « défini par les relations de voisinage entre points ou éléments » (p. 753). Les utopies et les hétérotopies constituent alors des emplacements en relation avec tous les autres emplacements de la société, « sur un mode tel qu'ils suspendent, neutralisent ou inversent l'ensemble des rapports qui se trouvent, par eux, désignés, reflétés ou réfléchis » (p. 756).

Si les utopies sont des «emplacements sans lieu réel »(p. 755), les hétérotopies se présentent pour leur part comme

des lieux réels $[\ldots]$ et qui sont des sortes de contre-emplacements, sortes d'utopies effectivement réalisées dans lesquelles les emplacements réels [...] sont à la fois représentés, contestés et inversés, des sortes de lieux qui sont hors de tous les lieux, bien que pourtant ils soient effectivement localisables (p. 755-756).

Les JRPEV goréens contestent en creux l'espace social contemporain de la surmédiation en marge duquel ils se situent lorsqu'ils se présentent comme lieu de traduction du fantasme de démédiation. En ce sens, ils se rapprochent des hétérotopies dites de compensation, dans lesquels l'espace se veut « aussi parfait, aussi méticuleux, aussi bien arrangé que le nôtre est désordonné, mal agencé et brouillon» (p. 758).

\section{Le système d'ouverture et de fermeture des hétérotopies goréennes}

Foucault (1994) établit six principes hétérotopologiques en vue de décrire les espaces autres. Le cinquième principe nous apparaît fondamental dans le contexte des hétérotopies goréennes, puisque c'est en vertu de celui-ci que se dessine la relation dynamique qui oppose la démédiation et la surmédiation. Il stipule que les hétérotopies possèdent un système d'ouverture et de fermeture qui les isole et les rend pénétrables. En ce qui concerne les hétérotopies goréennes, ce système régit non seulement l'accès des individus, mais également des textes exogènes. Ce système se distribue entre les modes de circonscription, de mise en relation et de mise en coprésence. 


\section{Le mode de circonscription}

La source fondamentale de la forme de l'utopie, écrit Jameson (2007), est " la nécessité formelle de la clôture utopique " (p. 357). Nous avons vu précédemment que cette clôture était mise à mal, au plan spatial, par la qualité réticulaire des hétérotopies goréennes. Les By-the-Books mobilisent néanmoins d'autres formes de clôture dans leurs tentatives de préserver l'intégrité de l'ordre naturel, qui, rappelons-le, représente une traduction particulière, dans les JRPEV goréens, de l'élan utopique qu'est le fantasme de démédiation.

Parmi ces formes de clôture, certaines sont de nature réglementaire et se caractérisent par une relative inflexibilité. À l'inverse, il existe des types de clôture flexibles : l'ouverture des hétérotopies cache alors une forme obreptice de fermeture et de circonscription. Sans présenter ici de typologie exhaustive des différents modes de circonscription, nous présenterons les plus importants dans leur application par les joueurs By-the-Books.

\section{Clôtures inflexibles}

Les clôtures réglementaires (inflexibles) relèvent d'une logique binaire : l'accès à un sim (espace virtuel en trois dimensions) est permis ou refusé. Le non-respect des règlements entraîne l'expulsion ou le bannissement des joueurs fautifs et l'invalidation de leurs actions. Parmi ces règlements, certains ont pour fonction de maintenir l'intégrité du thème goréen et, corrélativement, les conditions de possibilité d'une utopie de la démédiation. C'est le cas de ceux qui fixent le degré de familiarité des joueurs envers le monde de Gor et les Chroniques de Gor: des connaissances insuffisantes peuvent suffire à empêcher un joueur d'intégrer un sim By-the-Books. C'est également le cas des exemples suivants : ainsi, le règlement $1.1 \mathrm{du}$ sim City of Treve stipule que seuls les tenues et les armements goréens sont acceptés, lesquels s'inspirent de la culture médiévale, et le règlement 1.2, que les avatars non-humains, les esprits et les morts-vivants ne sont pas admis (Gor-SL, 2011). Quelques-uns de ces règlements concernent plus spécifiquement l'ordre naturel, suivant lequel les femmes ne peuvent faire montre de compétences martiales équivalentes à celles de leurs vis-à-vis masculins. Le règlement 2.e du sim The Great Tahari rappelle cette prescription lorsqu'il précise que les femmes ne peuvent porter d'armes, à l'exception de dagues courtes.

\section{Clôtures flexibles}

Le principe de la clôture flexible témoigne des stratégies présentes dans un jeu en vue de circonscrire une posture interprétative donnée sur une thèse (une doctrine) voulue non ambiguë, monosémique (Duret 2014b). Circonscrire et non imposer : le principe de clôture ainsi compris ne constitue pas un acte frontal de persuasion, mais un acte plus subtil conduisant à estomper certaines interprétations, à accompagner le joueur sur ce qui a l'apparence d'un territoire 
vierge et foisonnant et qui prend en réalité la forme d'un sentier obrepticement balisé (Duret 2015).

Parmi ces modes de clôture, les premiers sont d'ordre herméneutique et se rapportent à la signification. La clôture herméneutique vise à promouvoir une perspective particulière sur la loi de l'ordre naturel et, corrélativement, renforce le fantasme de démédiation. Il s'agit de protéger cette loi et ce fantasme des interprétations concurrentes susceptibles de les remettre en question ou de les subvertir. Elle passe par les activités exégétiques des joueurs, manifestations à partir desquelles une interprétation personnelle des romans de Norman est mise en avant. Ces exégèses sont diffusées sur des forums, des blogues, des encyclopédies en ligne et des aide-mémoire. Leur influence sur les jeux de rôle goréens est grande, puisque nombre de joueurs n'ont pas lu les Chroniques de Gor et connaissent le monde de Gor par l'intermédiaire de ces exégèses exclusivement, ces dernières leur servant d'auxiliaire dans la création de leur personnage. Ils adoptent donc la perspective des exégètes sans être en mesure d'élaborer leur propre interprétation, perspective qui influence les séances de jeu dans lesquelles ils sont impliqués.

D'autres modes de clôtures flexibles ont pour tâche de circonscrire le champ expérientiel des joueurs en limitant stratégiquement le spectre des actions des avatars (combats, échanges érotiques, etc.). Ces modes se présentent notamment sous la forme de scénarios proposés aux joueurs en vue de structurer leur jeu de rôle, d'affordances ${ }^{2}$ inscrites dans les paysages et l'architecture des sims et de dispositifs techniques. Ces modes prennent une forme indirecte de persuasion, puisqu'il n'est pas question de convaincre de la validité d'une thèse mais de conduire les joueurs à adopter un type d'expériences vidéoludiques inscrits dans la logique d'une thèse aux dépens d'autres expériences. Parmi ces modes, nous décrirons la clôture sociotechnique (Duret 2014b), qui décrit les interprétations de la loi de l'ordre naturel inscrites à même les dispositifs techniques des JRPEV goréens ou, plus précisément, à même les règles informatiquement implémentées qu'ils ont à charge d'appliquer et qui génèrent un champ d'actions possibles.

$\mathrm{Au}$ nombre des dispositifs sociotechniques ouvrant à la clôture d'une utopie médiévale de la démédiation figurent le G\&S, le Gorean Meter Craft Hud et le Nutri Life System. Tous trois contribuent à simuler un champ des actions possibles favorable à l'expérimentation d'une condition démédiée. Ainsi, une économie prémoderne et un rapprochement vers l'environnement (voire un volontaire état de dépendance envers celui-ci) sont proposés aux joueurs à l'aide de ces dispositifs : sous forme de matériaux bruts, la nature est façonnée par des artisans qui produisent leurs propres biens (armes, concoctions médicinales, outils, etc.). Elle est également consommée en tant que produit de la chasse, de la cueillette, de l'élevage et de l'agriculture.

2 Caractéristiques d'un objet suggérant certaines actions spécifiques à celui qui s'en sert en raison de sa configuration. 


\section{Un système de mise en relation}

Le système d'ouverture et de fermeture des hétérotopies goréennes contribue à la construction d'une utopie de la démédiation sur le mode de la clôture. Il y concourt également sur le mode de la mise en relation, au plan de l'architextualité, notamment, mais suivant un principe d'ouverture, cette fois-ci.

Genette (1982) définit l'architextualité comme « l'ensemble des catégories générales, ou transcendantes - types de discours, modes d'énonciation, genres littéraires, etc. - dont relève chaque texte singulier» (p. 7). Dans le cadre des JRPEV goréens, une part du phénomène d'architextualité est signalée lorsqu'il $\mathrm{y}$ a migration de tropes, de thèmes, de motifs et de topoï porteurs du fantasme de démédiation. Cette migration assure une redondance de cet élan utopique et traduit en même temps une ouverture du texte vidéoludique sur un vaste corpus de textes exogènes issus de diverses catégories transcendantes : œuvres issues des genres de la science-fiction (en particulier la romance planétaire) et de la fantasy, tous médias confondus.

Un exemple d'architextualité est le thème de la stase technologique. On le retrouve dans plusieurs œuvres de science-fiction telles que Dune, de Frank Herbert, où, à la suite d'une guerre entre humains et machines (le Jihad butlérien), l'ordinateur est proscrit et remplacé par des mentats, des individus aux capacités computationnelles surdéveloppées. Dans les Chroniques de Gor et les JRPEV, la stase technologique résulte d'un interdit religieux promulgué par les Prêtres-rois, les dieux goréens. Sans cette stase, les conditions d'un mode de vie prémoderne et d'une application du fantasme de démédiation ne sont pas rencontrées.

La mise en relation agit non seulement au plan textuel, mais également au plan spatial. Cette fois-ci, l'ouverture engendre de l'ambiguité et non une forme de clôture. Les hétérotopies goréennes sont traversées par deux modes de configuration spatiale concurrents : un espace de localisation et un espace de l'emplacement. Le fantasme de démédiation se présente notamment comme la recherche d'un espace social hiérarchisé et ordonné au sein duquel les individus occupent une position claire et entretiennent des relations interpersonnelles concrètes - espace non ambigu qui caractérise les JRPEV goréens, avec leur système de castes, leur code du guerrier et leurs prescriptions rigides au sujet des relations hommes-femmes. Cet espace ordonné, mimétique de l'espace de localisation du Moyen Âge décrit par Foucault, s'oppose à l'espace contemporain réticulaire, au sein duquel les relations sociales apparaissent comme complexes, abstraites et ambigües.

L'insularité est une caractéristique de l'utopie, nous dit Pagès (2000), une île constituant un espace isolé et protégé du monde qui est critiqué en creux. Elle est partagée par les sims goréens et les sims de Second Life en général, le MUVE californien se présentant sous la forme d'un vaste archipel d'espaces interreliés. L'insularité est concordante avec la constitution d'un espace- 
refuge prémoderne, une enclave de démédiation au cœur de la surmédiation contemporaine et s'inscrit dans la logique de fermeture des hétérotopies goréennes. Mais si la configuration insulaire de l'espace favorise l'isolement, il n'en demeure pas moins une relation privilégiée entre ces îles goréennes et le continent que constitue la société contemporaine, en raison d'une forme de critique passive : l'élan utopique du fantasme de démédiation est en soi une mise en procès de cette société surmédiée qu'elle rejette tout en y étant irrémédiablement liée sur le mode de la (non-) référence. Déjà, une brèche peut être observée. Mais ce n'est pas tout : d'une part, chaque sim goréen est accessible à l'aide de coordonnées (une adresse SLURL) qui, une fois entrées dans le navigateur de Second Life, permettent au joueur de s'y téléporter. Dès lors, il figure au cour d'une vaste toile hypertextuelle. D'autre part, il existe un sim, le Gor Hub, qui permet aux joueurs de rallier l'ensemble des autres sims goréens. Il propose aux joueurs une carte interactive de Gor sur laquelle il suffit de cliquer pour atteindre le sim voulu. Ces deux exemples sont représentatifs de l'emplacement, " défini par les relations de voisinage entre points ou éléments » (Foucault 1994 : 753).

Une relation dynamique s'établit donc clairement entre un espace de la localisation (un enclave d'ordre, de fermeture et de repli) et un espace de l'emplacement (réticulaire, décloisonné et acentrique), relation qui exemplifie ces utopies post-modernes que Jameson (2007) qualifie d' " îles en réseau, une constellation de centres discontinus, eux-mêmes décentrés » (p. 373).

\section{Un système de mise en coprésence}

En vertu du troisième principe de l'hétérotopologie foucaldienne, une " hétérotopie a le pouvoir de juxtaposer en un seul lieu réel plusieurs espaces, plusieurs emplacements qui sont en eux-mêmes incompatibles " (Foucault 1994 : 758). C'est le cas du cinéma et du théâtre, notamment. Suivant le sixième principe, une hétérotopie remplit une fonction : soit de compensation, soit d'illusion. Dans les deux cas, il y a contestation de l'espace restant. Si ces fonctions sont vues par le philosophe comme deux pôles extrêmes, elles sont coprésentes dans les hétérotopies goréennes. Nous avons vu précédemment ce qu'elles retiennent de la compensation. Les hétérotopies relevant du second pôle " ont pour rôle de créer un espace d'illusion qui dénonce comme plus illusoire encore tout l'espace réel » (p. 761). Les hétérotopies goréennes constituent un univers à la fois simulé et fictionnel. En ce sens, elles constituent une construction artificielle et une illusion. Ce ne sont pas les joueurs mais leurs personnages qui vivent des péripéties dans le monde de Gor. Or, cet univers repose sur le principe d'une relation à l'environnement physique démédiée, mise en scène dans un espace virtuel représentatif de la surmédiation contemporaine (ici, un excès de symbolisation) lui-même 
émaillé de socialités ${ }^{3}$ propres à la surmédiation contemporaine, comme nous le verrons plus bas. Il s'agit bien d'une illusion dénonçant comme illusoire l'espace réel. En accord avec le sixième principe, les hétérotopies goréennes remplissent à la fois une fonction de compensation et une fonction critique. En ce qui a trait à la fonction critique, on tente de combler un désir suscité par un état de société jugé insatisfaisant. En ce qui a trait à la fonction critique, ensuite, on juxtapose des rapports à l'environnement et des configurations sociales conflictuelles, voire incompossibles.

Voyons maintenant comment les hétérotopies goréennes juxtaposent des espaces incompatibles et en quoi cela relève d'un système d'ouverture : un réseau de points de vente émaille l'ensemble de l'archipel de Second Life, sims goréens compris. Il peut être vu comme un non-lieu ${ }^{4}$ (ou espace atopique) représentatif de l'économie de l'immatériel et permet la vente d'accessoires et de dispositifs techniques destinés aux avatars des joueurs, rendant le jeu possible dans les JRPEV. Les points de vente nécessitent des paiements en dollars Linden, la devise virtuelle de Second Life. Celle-ci est intégrée à l'économie réelle, toutefois, puisqu'elle est convertible en devises réelles. Parallèlement, des dispositifs techniques, le GESS, par exemple, sont élaborés en vue d'enrichir le jeu de rôle et simulent une économie prémoderne axée sur l'artisanat, l'élevage et l'agriculture. Cependant, les biens produits sont susceptibles d'être vendus en échange de dollars Linden, devise en prise, nous l'avons vu, sur l'économie réelle. En conséquence, certains joueurs génèrent des revenus en devises réelles par la vente de biens virtuels, au point où, si l'on se fie au témoignage de joueurs (Gor-SL 2015), certains maîtres profitent du travail gratuit de leurs esclaves pour produire des biens en série dans ce qui a tout l'apparence de manufactures modernes. Non seulement différents espaces économiques sont-ils convoqués, mais ils interagissent entre eux, la logique de démédiation du premier étant subvertie par la logique de surmédiation du second. Ici, l'ouverture fonctionne selon une logique de commutation entre deux niveaux du jeu qui, normalement, ne devraient pas se rencontrer : des dispositifs appartenant aux modalités de fonctionnement du jeu et un univers fictionnel.

\section{Conclusion}

Lorsqu'ils se déploient comme espace de localisation, se laissent émailler de tropes, de thèmes, de motifs et de topoï prémodernes, érigent des frontières les isolant d'un monde extérieur surmédié en vue de suggérer une vision alternative du vivre-ensemble et d'une relation à la nature reposant sur les principes de la loi de l'ordre naturel, les JRPEV goréens se présentent comme les garants

\footnotetext{
3 Défini comme « une forme socialisée du réel [qui se réfère à] un espace, un temps, un être ensemble, un système de codes, un système de relations et d'interlocuteurs, un complexe de normes, d'idées » (Duchet et Maurus $2011: 19$ ).

4 Espace transitoire remplissant une fonction économique et consumériste et voué à la circulation des biens, des informations et des personnes (Augé 1992).
} 
d'une condition utopique. En ce sens, ils construisent un éthos témoignant auprès des joueurs de la désirabilité de l'expérience d'une démédiation. Toutefois, le système d'ouverture et de fermeture assurant la viabilité d'une telle expérience compensatrice favorise dans le même mouvement un retour du refoulé : alors que l'utopie se réalise dans les sims de Second Life et se fait hétérotopie, elle se voit brouillée, contestée et subvertie par un ensemble de socialités propres à la surmédiation, présentes sur les modes de la mise en relation et de la mise en coprésence. Ainsi, l'espace réticulaire contemporain refuse à l'espace de localisation prémoderne toute cohérence - cette cohérence interne que l'on attend des mondes fictifs - lorsqu'il le contamine de socialités contemporaines surmédiées, atopiques (c'est-à-dire relevant du non-lieu). Ce faisant, la fonction critique de l'hétérotopie devient évidente, dans la mesure où s'affrontent au milieu de la même arène vidéoludique des socialités propres à la démédiation et à la surmédiation, et que cet affrontement allégorise, en quelque sorte, lomniprésence des réseaux atopiques contemporains, l'inévitabilité de la surmédiation et l'impossibilité de l'existence d'espaces refuges démédiés.

Le constat que nous venons de dresser témoigne de la relation à la fois intime et problématique que l'utopie nostalgique - lieu autre situé à la fois dans le passé et hors du temps, c'est-à-dire dans un passé idéalisé, voire mythique - entretient avec les lieux et le temps présents lorsqu'elle se réalise sur le mode virtuel, simulationnel. Qui plus est, ce constat valide la pertinence de cette hétérotopologie que proposait Foucault en son temps et dont les outils d'analyse restent à élaborer aujourd'hui.

\section{BIBLIOGRAPHIE}

AUGÉ Marc (1992), Non-lieux : Introduction à une anthropologie de la surmodernité, Paris, Seuil.

BOGOST Ian (2008), "The Rhetoric of Video Games », in K. Salen (dir.), The Ecology of Games : Connecting Youth, Games, and Learning, Cambridge, The MIT Press, p. $117-140$.

DUCHET Claude et Patrick MAURUS (2011), Un cheminement vagabond: Nouveaux entretiens sur la sociocritique, Paris, Honoré Champion.

DURET Christophe (2014a), "Living the Phantasm of Demediation : The Priest Kings and the Technology Prohibition in the Gorean Role-Playing Games », Heidelberg Journal of Religions on the Internet, Issue 7, p. 41-60.

DURET Christophe (2014b), « Strategies and Tactics in Digital Role-Playing Games: Persuasion and Social Negotiation of the Natural Order Thesis in Second Life's Gorean Community », in D. Ruggiero (dir.), Cases on Societal Effects of Persuasive Games, Hershey, IGI Global, p. 250-269. 
DURET Christophe (2015), "Five More Steps and You Will Be a New Person : The Procedural Closure of the Experiential Field in Every Day the Same Dream ", Comunicação e Sociedade, vol. 27, p. 95-114.

FOUCAULT Michel (1994), « Des espaces autres », Dits et écrits IV (1980-1988), Paris, Gallimard, p. 752-762.

FUNCKE Hans-Günter (1988), «L'évolution sémantique de la notion d'utopie en français ", in H. Hudde et P. Kuon (dir.), De l'utopie à l'Uchronie: Formes, Significations, Fonctions, Tübingen, Gunter Narr Verlag, p. 18-37.

GALLOWAY Alexander (2006), Warcraft and Utopia, CTheory.net. Disponible sur : http://www.ctheory.net/articles.aspx?id=507

GENETTE Gérard (1982), Palimpsestes : la littérature au second degré, Paris, Seuil.

Gor-SL. (2011), LAWS of TREVE revised 29th January 2011. Disponible sur : http://www.gor-sl.com/index.php?topic=748.0

Gor-SL. (2015), Using the G\&S roleplay system 2015. Disponible sur : http://www.gor-sl.com/index.php?topic=14817.0

JAMESON Fredric (2007), Archéologie du futur, tome 1 : Le désir nommé utopie, Paris, Max Milo.

PAGÈS Dominique (2000), « Des mondes parfaits aux mondes possibles : les territoires équivoques de l'utopie », Quaderni, Issue 41, p. 43-63.

SCHULZKE Marcus (2014), "The Critical Power of Virtual Dystopias », Games and Culture, vol. 9, Issue 5, p. 315-334.

SÉGUY Jean (1971), "Les sociétés imaginées : monachisme et utopie », Annales : Économies, Sociétés, Civilisations, vol. 26, Issue 2, p. 328-354.

Résumé : Cet article porte sur la construction d'une utopie prémoderne mue par un fantasme de démédiation et sa subversion par une logique de surmédiation contemporaine dans le cadre des jeux de rôle goréens de Second Life. Le concept d'hétérotopie, de Michel Foucault, sera mobilisé dans le but d'appréhender les modalités concrètes de cette tension. Le rôle joué par le système d'ouverture et de fermeture des hétérotopies goréennes sera examiné plus spécifiquement ici.

Mots-clés : eutopie, hétérotopie, jeux de rôle participatifs en environnement virtuel, JRPEV, utopie.

\begin{abstract}
This paper explores the construction of a premodern utopia driven by a phantasm of demediation and its subversion by the logic of contemporary overmediation in the context of the Second Life's Gorean role-playing games. The concept of heterotopia, elaborated by Michel Foucault, will be used with the aim of apprehending the concrete modalities of this tension. The role played by the opening and closure system of the Gorean heterotopias will be more specifically examined.
\end{abstract}

Keywords : eutopia, heterotopia, participatory role-playing games in virtual environment, PRPG-VEs. 